\title{
GESTÃO AMBIENTAL LOCAL: ANÁLISE DOS ARRANJOS PRODUTIVOS LOCAIS DE ALAGOAS UTILIZANDO O ÍNDICE MUNICIPAL DE GESTÃO AMBIENTAL
}

Márcio Jorge Porangaba Costa ${ }^{1}$

Michelle Cristiane de Lima Nunes ${ }^{2}$

Maria Cecília Junqueira Lustosa ${ }^{3}$

\section{Resumo}

A partir de 2000, foram formuladas políticas de fomento ao desenvolvimento, com base em Arranjos Produtivos Locais (APL), visando à geração de emprego e renda. Esses arranjos geram impactos localizados, muitos irreversíveis ou com altos custos de recuperação. Este trabalho tem por objetivo apresentar a metodologia do Índice Municipal de Gestão Ambiental (IMGA) e aplicá-lo aos municípios alagoanos que integram os APL. Conclui-se que, dentre os indicadores, a Agenda 21 não tem impacto significativo sobre o IMGA, embora estimule o município a criar mecanismos de desenvolvimento sustentável. A análise estatística mostra o nível insatisfatório dos índices que compõem o IMGA.

\footnotetext{
${ }^{1}$ É mestre em Desenvolvimento e meio ambiente pela Universidade Federal de Alagoas (UFAL), é professor adjunto da UFAL, e-mail: marcioporangaba@yahoo.com.br.

2 É mestre em Economia Aplicada da UFAL, e-mail: mlimanunes@gmail.com.

${ }^{3}$ É doutora em Economia da Industria e da Tecnologia pela Universidade Federal do Rio de Janeiro (UERJ), é professora associada da UFAL, e-mail: cecilialustosa@hotmail.com.
} 
Palavras Chaves: Gestão Ambiental. Arranjos Produtivos Locais. Sustentabilidade.

\begin{abstract}
The stimulation development policies based on Local Productive Arrangements (APL) has been made since 2000, aiming at employment and income improve. Those arrangements are responsable only for local impacts, many of them are irreversible or they can create high costs on recovery. This paper aims to present the Index of Municipal Environmental Management (IMGA) methodology, and show how to apply it to the municipalities that integrate the Alagoas APL. We conclude that among the indexes, the one that has been created based on Agenda 21 has no significant impact on the IMGA, although works as a encouragement tool to the public administration creates sustainable development mechanisms. Statistical analysis showed us an unsatisfactory level of conclusive responses for the index that compound the IMGA.
\end{abstract}

Keywords: Environmental Management. Local Productive Arrangements (LPA). Sustentability.

Classificação JEL: R11, R13 E R15

\title{
I Introdução
}

O desenvolvimento é um conceito que se amolda a interesses diversos, fazendo com que o planejamento com vistas à sua efetivação apresente especificidades em sua trajetória. O enfoque de redução ou eliminação da pobreza, da desigualdade e do desemprego de economias em crescimento foi característico das estratégias, nos anos de 1960. Nas duas décadas seguintes, iniciou a integração entre aspectos econômicos e sociais do desenvolvimento, enfatizando uma abordagem unificada para análise e planejamento do desenvolvimento que envolvesse todas as esferas da vida econômica e social.

Como consequência do debate suscitado em anos anteriores, nos anos de 1990, surge o novo ethos desenvolvimentista, que explicita o debate acerca da gestão de espaços territoriais e virtuais com vistas à promoção de ações orientadas para o desenvolvimento. Trata-se de uma nova aborda 
gem: a do desenvolvimento local, integrado e sustentável, com orientação para a competição e para a cooperação ou solidariedade.

No que tange à orientação para a competição, enfatiza-se a dimensão econômica do desenvolvimento ou a articulação entre agentes de desenvolvimento, atividades inovadoras e tecnologias de gestão social. Os seus protagonistas são governos, empresas, instituições, comunidades organizadas e redes produtivas que formam interorganizações configuradas a exemplo do entorno territorial inovador. A sua consolidação se dá por meio de enfoques em Arranjos Produtivos Locais (APL), ${ }^{4}$ Parques Tecnológicos, Incubadoras de Empresas, Clusters, entre outras estratégias. (LAGES; TONHOLO, 2006)

Na outra vertente, da orientação para a cooperação ou solidariedade, a ênfase é a inclusão de setores marginalizados na produção e no usufruto dos resultados, o que pressupõe a utilização de formas de produção não capitalistas e estratégias econômicas autônomas com tecnologias apropriadas. Entre os modelos adotados destacam-se: formas associativas de produção promovidas por instituições diversas, dinâmicas locais de orientação ecológica e sustentável, mecanismos de concessão de crédito e movimentos sociais, em geral. (SINGER; SOUZA, 2000)

Desta forma, o movimento de olhar o território como fator de competitividade, e não apenas como oferta de infraestrutura, começou a ser percebido, em diversas regiões do mundo, como uma forma convergente de integração e complementaridade de processos produtivos e de envolvimento de pequenos negócios em um contexto de encadeamento empresarial. (MELO; HANSEN, 2007) A partir das lógicas da cadeia produtiva e da competitividade integral - sistêmica setorial e empresarial - forma-se um microambiente ou local de Desenvolvimento Sustentável. (SACHS, 2004)

A nova territorialidade da política baseia-se no fato de que diferentes escalas geográficas - cidades, regiões e Estados-Nação - se encontram em concorrência para um clima favorável aos negócios, razão pela qual o poder sobre recursos materiais e institucionais passa a situar-se não mais em instâncias globais ou locais, mas naqueles atores dotados de maior mobilidade espacial e de maior capacidade de efetuar o que vem a ser uma política de escala. (VALE, 2007)

\footnotetext{
4 "Arranjos Produtivos Locais são aglomerações territoriais de agentes econômicos, políticos e sociais, com foco em um conjunto específico de atividades econômicas e que apresentam vínculos e interdependência. Geralmente, envolvem a participação e interação de empresas - que podem ser consultoria e serviços, comercializadores, clientes, entre outros - e suas variadas formas de representação e associação. Incluem, também, diversas outras instituições públicas e privadas voltadas para: formação e capacitação de recursos humanos, como escolas técnicas e universidades; pesquisa, desenvolvimento e engenharia; políticas, promoção de financiamento." (LASTRES et al., 2002, p. 13)
} 
Nesta perspectiva, a análise dos condicionantes e desdobramentos territoriais de Arranjos Produtivos Locais (APL) em regiões específicas pressupõe considerar o território como espaço de reprodução dos grupos sociais, no sentido de que as relações sociais são espacial e geograficamente medidas. Trata-se, pois, de uma noção mais ampla de território, que se confunde com a noção de espaço geográfico.

Os APL podem contribuir de maneira significativa para a efetivação do desenvolvimento, desde que não se constituam em objetivos de políticas em si, mas como uma ferramenta que promove uma visão sistêmica, capaz de mobilizar meios e instrumentos para o desenvolvimento. Este deve ser visto como um processo de mudanças estruturais, gerando dinamismo econômico, inserção e empoderamento social e sustentabilidade ambiental, rumo ao Desenvolvimento Sustentável.

Desde o início dos anos 2000, diversos Estados brasileiros estabeleceram políticas de fomento ao desenvolvimento, com base em Arranjos Produtivos Locais (APL), visando à geração de emprego e renda, sobretudo para as Micro e Pequenas Empresas (MPE). A inserção social e a sustentabilidade ambiental estão presentes em todos os planejamentos governamentais de apoio aos APL.

Todavia, todos esses arranjos fomentados por políticas de governos geram impactos sociais e ambientais localizados, uma vez que toda atividade econômica causa danos ao meio ambiente, muitos irreversíveis ou com altos custos de recuperação, prejudicando as partes afetadas - sejam os ecossistemas, seja a população, pela redução da qualidade de vida, sejam as próprias atividades econômicas que passam a sofrer restrições ambientais legais. Os impactos ambientais assumem uma dimensão localizada importante, tanto na depleção de recursos naturais, quanto na poluição local.

Dados os atuais níveis de poluição, sejam locais ou globais, e a acelerada depleção dos recursos naturais, faz-se cada vez mais urgente a mudança do atual padrão tecnológico para um padrão mais ecologicamente correto, por meio da adoção de inovações ambientais, que incluem aspectos organizacionais, produtivos, mercadológicos e tecnológicos, a fim de minimizar os impactos ambientais das atividades econômicas.

Entretanto, nas políticas de APL não estão contidos indicadores que possam avaliar sua sustentabilidade social e ambiental, pois são privilegiados aspectos econômicos, como a geração de emprego, renda e produção. Assim, é possível encontrar indicadores para avaliação de $A P L$, no que tange a seus resultados (Serviço Brasileiro Apoio as Micro e Pequenas 
Empresas - SEBRAE/ Sistema de Informação da Gestão Estratégica Orientada para Resultados - SIGEOR), à consolidação da governança local, ao quociente locacional, ao número de empregos absoluto, à inserção competitiva do APL junto ao mercado, ao potencial inovativo da atividade, e à disponibilidade de instituições de apoio na região.

A inserção de indicadores de Desenvolvimento Sustentável na metodologia dos Arranjos Produtivos Locais constitui-se uma necessidade, a fim de que as atividades desenvolvidas não afetem os demais atores do arranjo ou sistema - seja por meio da poluição, por meio de responsabilização de danos ambientais ou por meio da exclusão social, entre outros.

É nesta perspectiva, pois, que se insere o presente trabalho, uma vez que se propõe a apresentar a metodologia de construção do Índice Municipal de Gestão Ambiental (IMGA) e aplicá-lo aos municípios alagoanos que integram Arranjos Produtivos Locais. Tais indicadores podem se constituir em ferramentas auxiliares no processo de planejamento e gestão ambiental, no âmbito destes municípios, pelo fato de evidenciarem as áreas de melhor ou pior desempenho relativo, apontar tendências e chamar a atenção para pontos fracos.

O trabalho está organizado em quatro seções, incluindo esta introdução. Na sequência, é apresentada a metodologia adotada, isto é, a fonte dos dados, a concepção do Índice Municipal de Desenvolvimento Ambiental e o tratamento estatístico. Na terceira seção, são apresentadas as análises estatísticas, e na quarta, as conclusões.

\section{2 Índice Municipal de Gestão Ambiental - IMGA}

A importância de construir um Índice Municipal de Gestão Ambiental reside no fato de que o local é o lugar onde efetivamente existem maiores condições para a contenção, a prevenção e a solução da maioria dos problemas socioambientais, o que evidencia a importância do envolvimento da administração municipal em ações que contemplem a questão ambiental. (PHILIPPI JUNIOR et al., 1999; GOLDMEIER, 2004) O que pressupõe, sobretudo, a criação e/ou implementação de um sistema de indicadores para a tomada de decisões, no planos horizontal e vertical, e sua inserção no processo de gestão ambiental, em conformidade com os princípios do desenvolvimento sustentável. (OLIVEIRA, CARVALHO, BARCELLOS, 2003; BOLLINGER; NETO SCANDAR, 2004; COSTA, 2006; INSTITUTO BRASILEIRO DE GEOGRAFIA E ESTATÍSTICA, 2005; JANNUZZI, 2003; QUIROGA, 2001; SCANDER NETO, 2006; BALLEN, 2006) 


\section{I Concepção}

A Agenda 21, no capítulo 40, chama a atenção para o fato de que, para se caminhar rumo a este desenvolvimento, necessário se faz que a informação chegue a todos, isto é, aos tomadores de decisão locais, nacionais e internacionais, às organizações de base e, enfim, a todos os atores da sociedade. ${ }^{5}$

Na verdade, uma vez que a complexidade das questões presentes em um processo de gestão dos espaços naturais e antrópicos pode ser apresentada de forma simples, transparente e acessível, através de indicadores adequados (JANNUZZI, 2003; OLIVEIRA, CARVALHO, BARCELLOS, 2003), este procedimento resultará no fato de que cidadãos e tomadores de decisão se conscientizem do quadro socioeconômico e ambiental que se apresenta.

Entretanto, a mensuração do desenvolvimento socioeconômico e ambiental depara-se com limitações teórico-metodológicas. (COSTA; LUSTOSA, 2007; BELLEN, 2006; SCANDER NETO, 2006) Neste sentido, não existe consenso na literatura sobre o ideal em termos de indicadores a serem usados nessa perspectiva: se indicadores analíticos, distribuídos por temas e subtemas relacionados ao desenvolvimento sustentável, ${ }^{6}$ a exemplo do que propõem as Nações Unidas, ou se indicadores comensuralistas ou sintéticos, ${ }^{7}$ como o Índice de Desenvolvimento Humano - IDHM (PROGRAMA DAS NAÇÕES UNIDAS PARA O DESENVOLVIMENTO, 1990) e o Índice de Sustentabilidade Ambiental - ISA (ENVIROMMENTAL SUSTAINABILITY INDEX, 2002; MARTINS; FERRAZ; COSTA, 2006) O consenso é quanto a suas principais características (Princípios de Bellagio) ${ }^{8}$

\footnotetext{
${ }^{5}$ Para uma visão geral sobre a Agenda 21, ver Barbieri (2002).

${ }^{6}$ Trata-se do marco ordenador adotado pelo Brasil para a construção de indicadores de desenvolvimento sustentável. (IBGE, 2005b)

7 "O enfoque comensuralista se baseia na agregação ou somatório de variáveis de diversos tipos, utilizando uma escala comum de valor para contabilizar. Dentro deste enfoque se encontram os que agregam índices e os que agregam mediante unidades monetárias, físicas ou energéticas, tais como o IDH e contas Econômico-Ambientais." (OLIVEIRA, CARVALHO, BARCELLOS , 2003)

${ }^{8} \mathrm{Na}$ perspectiva de discutir as experiências em vários países sobre a mensuração do desenvolvimento sustentável e da sustentabilidade ambiental, um grupo de pesquisadores reuniu-se, em novembro de 1996, na Fundação Rockfeller, em Bellagio, na Itália. Na ocasião, foram aprovados os chamados Princípios de Bellagio, ou seja, foram definidos tópicos para a avaliação do progresso, no que diz respeito ao desenvolvimento sustentável, objetivando racionalizar e organizar centenas de indicadores, sobretudo os ambientais. Tais princípios são os seguintes: relevância do indicador para a formação de políticas, sua simplicidade e validade, existência de séries temporais, disponibilidade de dados produzidos com baixo custo, habilidades para agregar informação, sensibilidade para detectar pequenas mudanças e confiabilidade. (CATIZZONE, 2004, p. 228)
} 
Neste trabalho, optou-se pela construção de um indicador comensuralista - o Índice Municipal de Gestão Ambiental (IMGA) - composto de três indicadores, como explícito no Quadro 1.

Quadro 1 - Indicadores Municipais de Gestão Ambiental

\begin{tabular}{|c|c|c|}
\hline INDICADORES & A QUE SE PROPÕEM & CÁlCULO \\
\hline $\begin{array}{c}\text { Indicador de } \\
\text { Disponibilidade } \\
\text { de Instrumentos } \\
\text { Administrativos para a } \\
\text { Gestão Ambiental (IDIA) }\end{array}$ & $\begin{array}{c}\text { Identificar a } \\
\text { disponibilidade no } \\
\text { município j de: Órgão } \\
\text { de Meio Ambiente } \\
\left(\mathrm{x}_{1}\right) \text {, Funcionário }(\mathrm{s}) \\
\left(\mathrm{x}_{2}\right) \text {, Terceirização }\left(\mathrm{x}_{3}\right) \text {, } \\
\text { Recursos Financeiros }\left(\mathrm{x}_{4}\right) \\
\text { e Legislação Ambiental } \\
\left(\mathrm{x}_{5}\right) .\end{array}$ & $\operatorname{IDIAj}=\sum_{5}^{5 i}=1 \times i$ \\
\hline $\begin{array}{c}\text { Indicador de } \\
\text { Disponibilidade de } \\
\text { Articulação Institucional } \\
\text { para a Gestão Ambiental } \\
\text { (IDAI) }\end{array}$ & $\begin{array}{l}\text { Caracterizar a existência } \\
\text { no município j de: } \\
\text { Conselho Municipal de } \\
\text { Meio Ambiente }\left(x_{1}\right) \text { e de } \\
\text { Convênios e Acordos } \\
\text { Administrativos }\left(x_{2}\right) \text {, bem } \\
\text { como a sua participação } \\
\text { em Consórcios e Comitês } \\
\text { de Bacias }\left(x_{3}\right) \text {. }\end{array}$ & IDAlj $=\sum_{5}^{5} i=1 \times i$ \\
\hline $\begin{array}{c}\text { Indicador de } \\
\text { Disponibilidade de } \\
\text { Agenda } 21 \text { Local (IDA21) }\end{array}$ & $\begin{array}{l}\text { Caracterizar a existência } \\
\text { no município j de: } \\
\text { Agenda } 21\left(\mathrm{x}_{1}\right) \text { e do } \\
\text { Fórum da A21 Local }\left(\mathrm{x}_{2}\right) .\end{array}$ & $\operatorname{IDA} 21=\sum^{2} \mathrm{i}=1 \mathrm{xi}$ \\
\hline $\begin{array}{c}\text { Índice Municipal de } \\
\text { Gestão Ambiental (IMGA) }\end{array}$ & $\begin{array}{l}\text { Caracterizar a gestão } \\
\text { ambiental no âmbito do } \\
\text { município j. }\end{array}$ & $\begin{array}{c}|M G A j=| D I A+|D A|+\mid D A 21 \\
3\end{array}$ \\
\hline
\end{tabular}

Fonte: Elaboração própria

O IMGA constitui-se em uma adaptação do Índice de Amadurecimento Gerencial, proposto por Menezes (2006), para os municípios do Estado do Rio de Janeiro, e contempla uma das dimensões do desenvolvimento sustentável - a dimensão institucional - com variação entre zero e um.

\subsection{Fonte de Dados}

A fonte dos dados utilizada neste trabalho foi o Suplemento de Meio Ambiente da Pesquisa de Informações Básicas Municipais (MUNIC), realizada em 2002, pelo IBGE (2005a). Trata-se de uma pesquisa inédita no País, que cobriu todos os 5.560 municípios brasileiros instalados até 
$1^{\circ}$ de janeiro de 2001, por abordar, não só temas específicos à área ambiental local, como também o estado do meio ambiente no município. O seu informante foi o gestor local, secretário ou responsável pelo setor ambiental na prefeitura.

Quanto à coleta de informações, esta ocorreu, basicamente, no primeiro semestre de 2003. Os dados referem-se, de maneira geral, à data de entrevista, excetuando-se: (1) o número de funcionários (31 de dezembro de 2002), (2) fontes de recursos financeiros (ano de 2001), (3) condições do meio ambiente ( 24 meses anteriores) e programas e ações ambientais (12 meses anteriores).

O Suplemento se estrutura em 11 blocos, com base no modelo PRESSÃO-ESTADO-RESPOSTA (PER), ${ }^{9}$ ou seja, visa obter informações sobre as causas dos problemas ambientais $(\mathrm{P})$, os respectivos problemas ambientais (E) e programas e ações, bem como a organização da prefeitura (R). Não se pode, no entanto, deixar de fazer referência as suas limitações (OLIVEIRA, CARVALHO, BARCELLOS , 2003), a saber: (1) a relação entre as variáveis é linear, não há múltiplas causalidades; (2) é difícil determinar a pressão e, sobretudo, a resposta para cada tema; (3) um indicador pode ser de estado ou pressão, a depender do tema; (4) é mais difícil de operacionalizar em termos de políticas públicas e (5) não define metas.

Além do mais, outros cuidados devem ser levados em consideração, quando da análise dos dados. Entre estes se destacam: questões objetivas versus questões subjetivas, população nos municípios, existência versus intensidade de problemas ambientais, número desigual de municípios por Estado, diferença entre os municípios em termos de área e população, existência versus efetivo funcionamento, a exemplo de órgão municipal de meio ambiente e respostas múltiplas. (OLIVEIRA, CARVALHO, BARCELLOS, 2003)

Convém lembrar também que a pesquisa em apreço possibilita vários recortes, ou seja, os municípios podem ser agrupados por Unidade da Federação, Região, Faixas de população, Bacia Hidrográfica, Bioma, entre outros, a exemplo do adotado neste trabalho: os municípios foram agrupados por Arranjos Produtivos Locais - ALP, alvo de políticas públicas do Estado de Alagoas, de acordo com composição determinada pelo Programa de Arranjos Produtivos Locais de Alagoas (PAPL), sob a coordenação da

\footnotetext{
${ }^{9}$ Este marco ordenador e suas derivações (Força Motriz-Estado-Resposta; Estado-Pressão-ImpactoResposta; Força Motriz-Pressão-Estado-Impacto-Resposta) foi adotado inicialmente pela Organização para a Cooperação e Desenvolvimento Economico (OCDE), para organizar suas estatísticas ambientais. (OLIVEIRA, CARVALHO, BARCELLOS, 2003)
} 
Secretaria Executiva de Planejamento do Governo do Estado (SEPLAN/AL) e outros parceiros, como o SEBRAE do estado de Alagoas.

Utilizou-se também o Índice de Desenvolvimento Humano Municipal (IDHM) calculado pelo INSTITUTO DE PESQUISA ECONÔMICA APLICADA (IPEA)/PROGRAMA DAS NAÇÕES UNIDAS PARA O DESENVOLVIMENTO (PNUD), referente a 2000, para evidenciar a existência ou não de correlação entre o Índice Municipal de Gestão Ambiental (IMGA) e o referido índice.

\subsection{Tratamento Estatístico dos Dados}

O tratamento dos dados foi dividido em duas etapas: na primeira etapa, elaborou-se uma análise descritiva de cada variável considerada no presente trabalho, no intuito de observar a variabilidade, distribuição e localização de seus valores. Para tanto, utilizou-se o software SPSS (versões 10 e 16), para obter medidas estatísticas de localização, como média, mediana e moda; estatísticas de distribuição, como desvio-padrão, e obter teste de normalidade de distribuição, a partir das medidas de assimetria e curtose.

Na segunda etapa, foi feita uma análise de correlação entre as variáveis independentes - IDIA, IDAI, IDA21 e IDHM - em relação ao índice estruturado IMGA, com base em diagramas de dispersões e matrizes de correlações. As matrizes foram geradas em dois grupos distintos: primeiro foi feita a correlação do IMGA com os índices que o compõem e, posteriormente, a correlação do IMGA com a variável exógena IDHM. Já os gráficos, por definição estatística, possibilita analisar pares de variáveis, sendo uma delas sempre presente (IMGA), em relação a uma outra independente. Todo esse processo é concluído, utilizando-se o mesmo software citado anteriormente.

$\mathrm{Na}$ terceira etapa, testou-se a confiabilidade do índice estruturado IMGA, a partir do Alpha de Crombach (BISQUERRA, SARRIERA, MARTÍNEZ, 2004), que calcula o valor exato da sua confiabilidade.

Na quarta etapa, fez-se uma análise regressiva em dois grupos: no primeiro grupo, a análise relaciona o IMGA aos índices que o compõem, no intuito de observar o valor de variação que cada índice provoca sobre o IMGA, quando eles variam em uma unidade, o poder de explicação estatística dos índices sobre o IMGA e o poder de explicação do modelo como um todo. Isto posto, foi observada uma inesperada importância de um dos índices e, então, foi feita uma nova regressão, porém, em forma 
de modelo simples, para verificar o exato poder de explicação de cada variável sobre o IMGA e qual seu coeficiente de variação.

No segundo grupo, a análise regressiva relaciona o IMGA com a variável exógena considerada IDHM, seguindo os mesmos objetivos das regressões precedentes.

Dessa forma, os indicadores observados nas saídas de regressão do SPSS são, respectivamente, os coeficientes de regressão $-\beta_{k^{\prime}}$ as estatísticas de Student $-\mathrm{t}$, o valor $-\mathrm{p}$, intervalos de confiança dos parâmetros, o coeficiente de determinação - $R^{2}$ e a estatística de Fisher - F.

\subsection{Descrição dos APL}

Para a análise estatística do IMGA dos municípios que compõem os APL de Alagoas, consideraram-se os arranjos alvo de políticas públicas pelo PAPL. ${ }^{10}$ Sua classificação é organizada de acordo com o setor econômico em que o arranjo está inserido. Seguindo essa classificação, mensuramos a existência de dez APL no Estado, distribuídos em oito setores econômicos.

Como o intuito do presente trabalho é apresentar metodologia de construção de indicadores municipais de gestão ambiental, além de confirmar sua aplicabilidade, a fim de que se tornem ferramentas auxiliares no processo de planejamento e gestão ambiental, faz-se necessário a análise estatística dos resultados agregados dos índices selecionados (IDIA, IDAI e IDA21), do índice estruturado (IMGA) e da variável IDHM - que, sendo considerada exógena, tem influência sobre o índice estruturado - em relação ao total de APL existentes no Estado.

Assim sendo, os índices e a variável exógena considerada, segundo a distribuição dos setores econômicos de Alagoas, encontram-se em anexo.

\section{Análise e Discussão dos Dados}

Neste item serão descritos e analisados o padrão de distribuição e as estatísticas desctitivas de todas as variáveis consideradas.

\section{I Análise Estatística Descritiva}

O índice IDIA segue distribuição normal (Quadro 2), bem como a sua recodificação (Quadro 3). Esta última, organizada em quatro categorias, para diminuir o intervalo de valores, mostra que sete dos dez APL (70\%)

\footnotetext{
${ }^{10} \mathrm{Em}$ 2009, o número de APL fomentados pelo Governo do Estado subiu para 14.
} 
inserem-se na categoria 2, ou seja, têm seus índices médios entre 0,30 e 0,59 , as demais categorias têm inserido apenas um $A P L$, representando cada uma apenas $10 \%$ da amostra.

\begin{tabular}{|c|c|c|c|c|c|c|c|c|}
\hline $\begin{array}{c}\text { ESTATÍSTICAS } \\
\text { DE } \\
\text { LOCALIZAÇÃO } \\
\text { E DISTRIBUIÇÃO }\end{array}$ & 訔 & 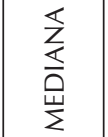 & $\begin{array}{l}\stackrel{\pi}{0} \\
\stackrel{0}{2}\end{array}$ & $\begin{array}{l}\text { INTERVALO DE } \\
\text { CONFIANÇA }\end{array}$ & $\begin{array}{l}\text { DESVIO- } \\
\text { PADRÃO }\end{array}$ & $\frac{0}{\substack{x \\
x}}$ & $\frac{\sum_{z}}{\sum}$ & $\sum_{0}^{\mathbb{K}}$ \\
\hline VALORES & 0,4630 & 0,4100 & $0,32 *$ & 0,$2912 ; 0,6348^{* *}$ & 0,2402 & 1 & 0,16 & 4,63 \\
\hline
\end{tabular}

Fonte: Elaboração própria

* Esse valor aparece duas vezes na amostra $=16,7 \%$.

** O intervalo foi construído para um nível de significância de 95\%.

Quadro 3 - Classificação da recodificação do IDIA

\begin{tabular}{|c|c|c|c|c|}
\hline CATEGORIAS & 1 & 2 & 3 & 4 \\
\hline INTERVALOS & $0,00-0,29$ & $0,30-0,59$ & $0,60-0,89$ & $0,90-1,00$ \\
\hline \multicolumn{5}{|c|}{ Fonte: Elaboração própria }
\end{tabular}

O que leva a concluir que o Indicador de Disponibilidade de Instrumentos Administrativos para a Gestão Ambiental se encontra em um nível relativamente crítico, ao estar muito próximo de zero na amostra selecionada.

O índice IDAI segue distribuição normal (Quadro 4), bem como a sua recodificação (Quadro 5). Esta última, organizada em quatro categorias, para diminuir o intervalo de valores, mostra que cinco dos dez APL (50\%) inserem-se na categoria 2, ou seja, têm seus índices médios entre 0,26 e 0,50 , seguidos de quatro deles $(40 \%)$, que se inserem na categoria 1 , ou seja, têm seus índices médios entre 0,00 e 0,25; a categoria 3 é responsável por um APL (10\%). Vale destacar que a categoria 4 não tem nenhum APL em sua composição, ou seja, nenhum APL tem um índice IDAI próximo de seu valor máximo 1 .

O que leva a concluir que o Indicador de Disponibilidade de Articulação Institucional para a Gestão se encontra num nível muito baixo, necessitando de atenção por parte das autoridades municipais. 


\begin{tabular}{|c|c|c|c|c|c|c|c|c|}
\hline $\begin{array}{c}\text { ESTATÍSTICAS } \\
\text { DE } \\
\text { LOCALIZAÇÃO E } \\
\text { DISTRIBUIÇÃO }\end{array}$ & 訔 & 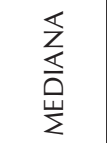 & $\begin{array}{l}\overleftarrow{0} \\
\stackrel{0}{0} \\
\stackrel{2}{2}\end{array}$ & $\begin{array}{l}\text { INTERVALO DE } \\
\text { CONFIANÇA }\end{array}$ & $\begin{array}{l}\text { DESVIO- } \\
\text { PADRÃO }\end{array}$ & $\stackrel{\substack{0 \\
x}}{\substack{x \\
\Sigma}}$ & 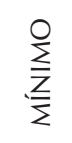 & $\sum_{0}^{\infty}$ \\
\hline VALORES & 0,3080 & 0,2900 & --** & 0,$1925 ; 0,4235 * *$ & 0,1614 & 0,60 & 0,07 & 3,08 \\
\hline
\end{tabular}

Fonte: Elaboração própria.

*A moda é considerada múltipla, uma vez que cada valor aparece na mesma quantidade (uma vez cada $=10 \%$ )

** O intervalo foi construído para um nível de significância de 95\%.

Quadro 5 - Classificação da recodificação do IDAI

\begin{tabular}{|c|c|c|c|c|}
\hline CATEGORIAS & 1 & 2 & 3 & 4 \\
\hline INTERVALOS & $0,00-0,25$ & $0,26-0,50$ & $0,51-0,75$ & $0,76-1,00$ \\
\hline
\end{tabular}

Fonte: Elaboração própria

O índice IDA21 segue distribuição normal (Quadro 6), mas sua recodificação (Quadro 7) não tem distribuição definida. Esta última, organizada em três categorias, para diminuir o intervalo de valores, mostra que todos os APL inserem-se na categoria 2, ou seja, tem seus índices médios entre 0,35 e 0,69 . Vale destacar que as demais categorias não tem nenhum APL em sua composição, ou seja, nenhum arranjo tem um índice maior que 0,00 e menor que 0,35 , como também, um índice IDA2 1 próximo de seu valor máximo 1.

\begin{tabular}{|c|c|c|c|c|c|c|c|c|}
\hline \multicolumn{9}{|c|}{ Quadro 6 - Estatísticas descritivas do IDA21 } \\
\hline $\begin{array}{c}\text { ESTATÍSTICAS } \\
\text { DE } \\
\text { LOCALIZAÇÃO } \\
\text { E } \\
\text { DISTRIBUIÇÃO }\end{array}$ & 离 & 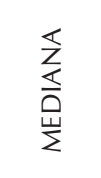 & $\begin{array}{l}\stackrel{1}{0} \\
\stackrel{0}{\Sigma}\end{array}$ & $\begin{array}{l}\text { INTERVALO DE } \\
\text { CONFIANÇA }\end{array}$ & $\begin{array}{l}\text { DESVIO- } \\
\text { PADRÃO }\end{array}$ & $\frac{\substack{x \\
\frac{x}{x}}}{\sum}$ & $\frac{\mathfrak{O}}{\sum}$ & 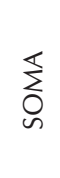 \\
\hline VALORES & 0,4460 & 0,4450 & $0,5^{*}$ & 0,$4114 ; 0,4806^{* *}$ & 0,0483 & 0,50 & 0,35 & 4,46 \\
\hline
\end{tabular}

Fonte: Elaboração própria

* Esse valor aparece três vezes na amostra $=30 \%$.

** O intervalo foi construído para um nível de significância de 95\%.

Quadro7 - Classificação da recodificação do IDA21

\begin{tabular}{|c|c|c|c|}
\hline CATEGORIAS & 1 & 2 & 3 \\
\hline INTERVALOS & $0,00-0,34$ & $0,35-0,69$ & $0,70-1$ \\
\hline
\end{tabular}

Fonte: Elaboração própria 
Isso leva a conclusão de que todos os municípios alagoanos que compõem os APL estão comprometidos num nível moderado com a proposta da Agenda 21 rumo a uma sociedade economicamente sustentável.

O índice IMGA segue distribuição normal (Quadro 8), mas, sua recodificação não (Quadro 9), servindo apenas como distribuição de frequência. Esta última, organizada em quatro categorias, para diminuir o intervalo de valores, mostra que nove dos dez APL $(90 \%)$ inserem-se na categoria 2, ou seja, têm seus índices médios entre 0,243 e 0,500, seguidos de um deles $(10 \%)$, que se insere na categoria 3 , ou seja, tem seus índices médios entre 0,501 e 0,700 .

Vale destacar que não há nenhum APL na categoria 1, ou seja, nenhum APL tem um IMGA menor que 0,242, mesmo tendo um de seus índices de composição igual a zero, como também não há nenhuma APL na categoria 4, ou seja, nenhum APL tem um IMGA maior que 0,700.

Porém, os índices ainda se encontram num nível relativamente baixo, considerando que quanto mais próximo de 1 , maior a qualidade de vida e mais próximo está de um desenvolvimento sustentável.

\begin{tabular}{|c|c|c|c|c|c|c|c|c|}
\hline \multicolumn{9}{|c|}{ Quadro 8 - Estatísticas descritivas do IMGA } \\
\hline \begin{tabular}{|} 
ESTATÍSTICAS \\
DE \\
LOCALIZAÇÃO \\
E \\
DISTRIBUIÇÃO
\end{tabular} & 岕 & 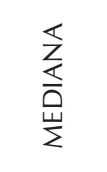 & $\begin{array}{l}\stackrel{\leftarrow}{0} \\
\stackrel{0}{0}\end{array}$ & $\begin{array}{l}\text { INTERVALO DE } \\
\text { CONFIANÇA }\end{array}$ & $\mid \begin{array}{l}\text { DESVIO- } \\
\text { PADRÃO }\end{array}$ & $\sum_{\substack{x \\
x}}^{0}$ & $\frac{\sum_{z}^{0}}{\frac{Z}{z}}$ & 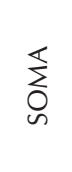 \\
\hline VALORES & 0,4023 & 0,3750 & $--*$ & 0,$3068 ; 0,4978 * *$ & 0,1333 & 0,700 & 0,243 & 4,023 \\
\hline
\end{tabular}

Fonte: Elaboração própria

* A moda é considerada múltipla, uma vez que cada valor aparece na mesma quantidade (uma vez cada $=10 \%$ )

** O intervalo foi construído para um nível de significância de 95\%.

Quadro 9 - Classificação da recodificação do IMGA

\begin{tabular}{|c|c|c|c|c|}
\hline CATEGORIAS & 1 & 2 & 3 & 4 \\
\hline INTERVALOS & $0,00-0,242$ & $0,243-0,500$ & $0,501-0,700$ & $0,701-1,000$ \\
\hline
\end{tabular}

Seguindo o mesmo raciocínio, a análise estatística descritiva da variável exógena considerada neste trabalho - IDHM - mostra que o IDHM não segue distribuição normal (Quadro 10) e nem a sua recodificação (Quadro 11) que, organizada em três categorias, para diminuir o intervalo de valores, mostra que $100 \%$ dos APL inserem-se na categoria 2, ou seja, têm seus valores médios entre 0,500 e 0,750. 
Quadro 10 - Estatísticas descritivas do IDHM

\begin{tabular}{|c|c|c|c|c|c|c|c|c|}
\hline $\begin{array}{l}\text { ESTATÍ́STICAS } \\
\text { DE } \\
\text { LOCALIZAÇÃO } \\
\text { E } \\
\text { DISTRIBUIÇÃO }\end{array}$ & 嵩 & $\begin{array}{l}\text { z } \\
\text { 文 } \\
\text { 岁 }\end{array}$ & $\begin{array}{l}\frac{\pi}{0} \\
\frac{0}{2}\end{array}$ & $\begin{array}{c}\text { INTERVALO } \\
\text { DE } \\
\text { CONFIANÇA }\end{array}$ & $\begin{array}{l}\text { DESVIO- } \\
\text { PADRÃO }\end{array}$ & $\frac{\sum_{x}^{0}}{\sum}$ & $\frac{\sum_{\Sigma}}{\frac{Z}{\Sigma}}$ & $\sum_{0}^{\infty}$ \\
\hline VALORES & 0,599 & 0,583 & $-{ }^{*}$ & 0,$559 ; 0,641 * *$ & 0,057 & 0,739 & 0,546 & 5,999 \\
\hline
\end{tabular}

Fonte: Elaboração própria

* A moda é considerada múltipla, uma vez que cada valor aparece na mesma quantidade (uma vez cada $=10 \%$ )

** O intervalo foi construído para um nível de significância de 95\%.

Quadro 11 - Classificação da recodificação do IDHM

\begin{tabular}{|c|c|c|c|}
\hline CATEGORIAS & 1 & 2 & 3 \\
\hline INTERVALOS & $0,000-0,500$ & $0,500-0,750$ & $0,750-1,000$ \\
\hline
\end{tabular}

Fonte: Elaboração própria

\subsection{Análise de Correlação}

O teste de correlação entre o índice estruturado e os índices que o compõem, baseado na estatística R de Pearson, mostra que:

1. há uma alta correlação entre IMGA e o IDIA, com uma estatística de Pearson de 0,979, erro-padrão de 0,015 (estatística $t=13,693$ ) e um valor-p de 0,000, mostrando com confiança de $95 \%$ que o eles estão correlacionados em cerca de $96 \% ;^{11}$

2. há uma alta correlação entre IMGA e o IDAI com uma estatística de Pearson de 0, 883, erro-padrão de 0,064 (estatística $t=5,319$ ) e um valor-p de 0,001 , mostrando com confiança de $95 \%$ que eles estão correlacionados em $78 \%$;

3. há uma fraca correlação entre o IMGA e o IDA21, com uma estatística de Pearson de 0,501, um erro-padrão de 0,168 (estatística $t=1,638$ ) e um valor-p de 0,140, mostrando com confiança de $86 \%$ - devido à probabilidade de erro de $14 \%$ - que eles estão correlacionados em $25 \%$.

No quadro de correlações dado pelo programa SPSS, o IMGA está correlacionado em mais de $70 \%$ com os índices IDIA e IDAI e em apenas $25 \%$ com o IDA21. Outra informação importante aparece no quadro de saída do SPSS: há uma correlação muito grande entre os índices IDIA e IDAI, podendo comprometer uma posterior análise múltipla.

\footnotetext{
${ }^{11}$ O percentual compreende o quadrado do coeficiente de correlação multiplicado por 100.
} 
Os diagramas de dispersões, testes de correlações e as regressões confirmam que a variável exógena IDHM influencia o IMGA.

A matriz de correlação mostra que o IMGA está altamente relacionado com o IDHM, em mais de $70 \%$. Os gráficos de dispersão também comprovam.

\subsection{Análise de Confiabilidade do IMGA}

Confirmada a correlação entre o IMGA e os índices que o compõem, faz-se necessário testar estatisticamente sua confiabilidade.

Para que haja consistência entre as variáveis componentes de um índice, o valor do Alpha de Crombach (BISQUERRA, SARRIERA, MARTíNEZ, 2004) deve ser maior que 0,7 . Esse valor é um percentual de confiabilidade do índice, portanto, quanto mais próximo de 1, melhor - mais confiável será o valor do índice que está para ser formado.

Os resultados do teste emitidos pelo programa SPSS mostram que o valor de Alpha do índice IMGA é de 0,7027 e 0,7344 padronizado. O mesmo também mostra que, se os índices IDIA ou IDAI deixarem de ser componentes do IMGA, o valor de Alpha se reduz consideravelmente, confirmando o teste de correlação acima, que mostra a alta relação entre esses índices e o IMGA. Seguindo o mesmo raciocínio, o resultado do programa mostra que, se o índice IDA21 deixar de compor o IMGA, este último terá um aumento de valor para 0,8516, confirmando sua pequena correlação.

Teoricamente, a Agenda 21 tem um grande impacto sobre o IMGA, no fato de estimular o município que se compromete com ela, a criar mecanismos de desenvolvimento sustentável, impactando o valor do IMGA. Por esse motivo, atribuímos a variação de sua força estatística - o aumento do Alpha, caso este índice seja deletado -, ao fato de que a maioria dos municípios não se comprometeu e desenvolveu mecanismos de desenvolvimento sustentável suficientes para mostrar o seu impacto, além do fato de se estar trabalhando com uma amostra consideravelmente pequena.

Por esse motivo, em caráter temporário e experimental, aceitaremos o nível do valor de Alpha de Crombach de confiabilidade do índice estruturado. 


\subsection{Análise de Regressão}

Estando verificada a correlação, entre os índices independentes e o índice dependente, e a confiabilidade do IMGA, segue-se com a mensuração individual dessa relação.

A análise de regressão múltipla entre essas variáveis selecionadas tem os resultados apresentados a seguir.

No quadro 1 da saída de regressão do SPSS tem-se o resumo dos valores dos coeficientes, seus respectivos desvios-padrão, estatísticas de significância individuais (t) e as probabilidades de erro (valor-p). Segundo esses dados, obtém-se o modelo:

\begin{tabular}{|c|c|c|c|c|}
\hline IMGA = & $-0,0337$ & 0,332 IDIA & 0,318 IDAI & $+\quad 0,414$ IDA21 \\
\hline Ep & $(0,022)$ & $(0,017)$ & $(0,023)$ & $(0,052)$ \\
\hline Est. $\mathrm{t}$ & $(-1,532)$ & $(19,012)$ & $(14,007)$ & $(7,889)$ \\
\hline $\mathrm{IC}^{12}$ & $(-0,088 ; 0,020)$ & $(0,289 ; 0,374)$ & $(0,262 ; 0,373)$ & $(0,286 ; 0,542)$ \\
\hline Valor-p & $(0,176)$ & $(0,000)$ & $(0,000)$ & $(0,000)$ \\
\hline
\end{tabular}

Esses dados dizem que, sem os índices que compõem o IMGA, não haveria tal índice, ou seja, não há um IMGA autônomo, pois o valor de seu coeficiente é teoricamente inaceitável, sua estatística de significância individual é estatisticamente insignificante, e seu valor-p confirma sua irrelevância, mostrando que a probabilidade real de cometer um erro, caso a aceite, é maior que o nível de significância adotado, de 5\%.

O valor do coeficiente do IDIA é teoricamente plausível e mostra que à medida que aumenta em uma unidade, eleva o valor de IMGA em 0,332. Essa relação é estatisticamente significante, com um valor da estatística t elevadíssimo, em 19,012, e um valor-p baixíssimo, em 0,000.

O valor do coeficiente do IDAI também é teoricamente plausível e mostra que à medida que aumenta em uma unidade, eleva o valor de IMGA em 0,318. Essa relação é estatisticamente significante, com um valor da estatística t elevadíssimo, em 14,007, e um valor-p baixíssimo, em 0,000.

$\mathrm{O}$ valor do coeficiente do IDA21 mostra que à medida que aumenta em uma unidade, eleva o valor de IMGA em 0,414. Essa relação é estatisticamente significante, com um valor da estatística t elevado, de 7,889, e um valor-p baixíssimo, em 0,000.

${ }^{12}$ Intervalo de Confiança de 95\%. 
É importante destacar que os valores t de IDIA e IDAI confirmam os ajustes desses índices em relação ao IMGA, ou seja, o IDIA tem maior poder de explicação que a IDAI. Já é surpreendente o valor da estatística t do IDA21, expressando maior poder de explicação que o apresentado nos testes de correlações anteriores.

O quadro 2 da saída de regressão do SPSS mostra o coeficiente de determinação do modelo que diz que os valores dos índices independentes (IDIA, IDAI e IDA21) explicam 99,9\% da variação do IMGA.

O quadro 3 da saída de regressão do SPSS mostra a divisão da explicação do modelo entre a regressão e o termo residual da Análise de Variância (ANOVA). Nela, podemos ver que, de um total de 0,160 de variação, a regressão é responsável pela explicação de 0,160, ou seja, 99,9\%; e para o termo residual é atribuído apenas 0,0002, ou seja, 0,1\% da explicação.

No intuito de confirmar ou corrigir essa surpreendente importância da variável IDA21, foram estimados modelos simples entre o IMGA e cada um dos índices que o compõem. Os resultados foram mais confiáveis, resultando nos seguintes modelos:

\section{Modelo 1:}

IMGA $=0,150+0,544$ IDIA

ep $(0,021)(0,040)$

est $t(7,327)(13,693)$

valor $\mathrm{p}(0,000)(0,000)$

IC $(0,103 ; 0,198)(0,453 ; 0,636)$

$\mathrm{R}^{2}=0,959 \mathrm{~F}=187,493$

\section{Modelo 2:}

IMGA $=0,177+0,73$ IDA

ep $(0,047)(0,137)$

est.t $(3,758)(5,319)$

valor $\mathrm{p}(0,006)(0,001)$

IC $(0,069 ; 0,286)(0,414 ; 0,047)$

$\mathrm{R}^{2}=0,78 \mathrm{~F}=28,287$ 
Modelo 3:

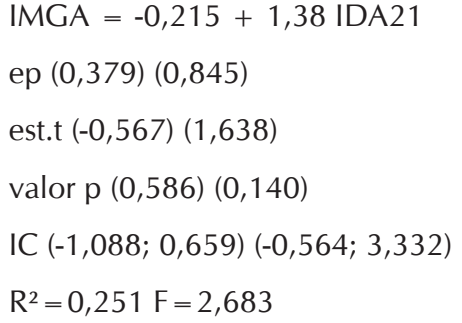

Esses modelos são mais confiáveis, pois expressam estatísticas mais coerentes com as análises estatísticas precedentes, inclusive no que se refere ao pequeno poder de explicação do IDA21 sobre o IMGA. Acompanhado os valores já mostrados anteriormente, estão o IC, intervalo de confiança, e a estatística F de significância global.

Como cada parâmetro está dentro dos seus respectivos intervalos de confiança, pode-se ter a certeza de que estaremos próximos do valor real em $95 \%$ dos casos. Já a estatística F testa a significância global do modelo e como em cada caso ela é maior que seu valor crítico de 1 grau de liberdade no numerador e 8 no denominador - exceto no modelo IMGA $=\mathrm{f}(\mathrm{IDA} 21)-$, concluímos que os dois primeiros modelos são estatisticamente confiantes em 95\% dos casos. Como esperado, esses valores não são tão altos para a estatística IDA21, cujas estatísticas de hipóteses, com valores menores que seus números críticos, mostram que o modelo não é confiável e que, caso o utilizemos para previsões, teremos uma margem de erro maior que $5 \%$.

Analisando regressivamente o IMGA em relação à variável exógena, obtivemos os resultados relatados no Modelo 4.

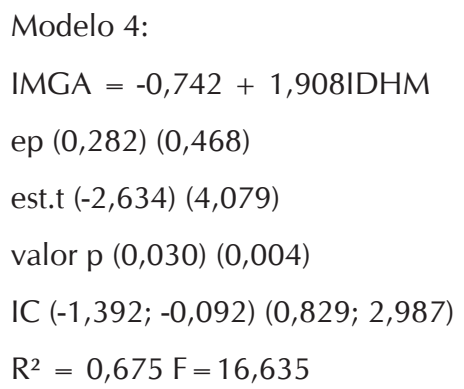

194 | Nexus Econômicos - CME-UFBA 
Esses resultados dizem que não há um índice de IMGA, caso a variável independente tome um valor zero. Também mostram que, à medida que o IDHM aumenta em 1 unidade, o IMGA aumenta em 1,908.

Esses valores têm suas importâncias comprovadas pelos seus valores da estatística t maiores que 2 e baixos valores-p. Os intervalos de confiança compreendem todos os valores dos seus respectivos parâmetros, dando- Ihes proximidade do verdadeiro valor, em $95 \%$ dos casos.

O coeficiente de determinação $\mathrm{R}^{2}$ diz que o modelo explica $67,5 \%$ da variação do valor do IMGA e a estatística de teste $\mathrm{F}$ confirma que o modelo é confiável ao nível de significância de $5 \%$, com 1 grau no numerador e 8 no denominador.

\section{Conclusão}

A proposta deste trabalho foi apresentar e aplicar a metodologia de construção de indicadores municipais de gestão ambiental - em particular nos municípios alagoanos que compõem APL - visando à detecção de um padrão de desempenho e tendências dos índices, interligado-os a outras variáveis, em âmbito municipal, no intuito de visualizar políticas públicas que promovam o desenvolvimento da economia local a um nível sustentável.

Nessa perspectiva, criou-se o Índice Municipal de Gestão Ambiental (IMGA), composto de três indicadores, a saber: Indicador de Disponibilidade de Instrumentos Administrativos para a Gestão Ambiental (IDIA), Indicador de Disponibilidade de Articulação Institucional para a Gestão Ambiental (IDAI) e Indicador de Disponibilidade de Agenda 21 Local (IDA21), com vistas a desenvolver um índice paramétrico de desenvolvimento sustentável influenciável por três outros índices de controle público municipal. A análise estatística de formação desse índice confirma sua confiabilidade, reforçando a grande participação dos três índices independentes no IMGA. A análise de correlação, por sua vez, comprova o grau de interação entre os índices independentes e o índice dependente, evidenciada também pelos valores pontuais da análise de regressão.

A análise estatística, em todos os seus âmbitos, mostra que os municípios alagoanos selecionados têm valores ainda insatisfatórios de seus índices (IDIA, IDAI e IDA21), chamando a atenção para a criação e/ou efetivação de políticas públicas que os fortaleçam, principalmente, ao saber que o fortalecimento dos mesmos elevará o valor do IMGA, provocando 
uma melhoria na qualidade de vida da população desses municípios. É importante também perceber que essa necessidade se faz mais urgente em alguns municípios do que em outros.

Uma vez estabelecido o poder de utilização do IMGA, outra proposta do trabalho foi criada: a necessidade de descobrir se e em que importância o mesmo (IMGA) se relaciona a outras variáveis de controle público municipal.

Nessa linha de raciocínio, o IMGA foi relacionado ao IDHM. As análises estatísticas em suas três visões, descritivas, de correlação e de regressão, confirmam a expectativa de que o investimento público em setores que compõem o IDHM (educação, saúde impactando na longevidade, e, na renda), provocará um aumento no índice estrutural, significando que a economia municipal segue rumo a um melhor nível de vida (sustentável).

A própria natureza do APL, gerando renda, conhecimento, inovação e, indiretamente, educação e saúde, eleva o IMGA, através da elevação do índice IDHM. Isso implica na necessidade de promoção de programas com vistas à construção de uma economia desenvolvida e sustentável.

Resta, pois, a conclusão de que políticas públicas no intuito de fortalecer os índices independentes (IDIA, IDAI e IDA21), principalmente os dois primeiros, a variável exógena, IDHM, através dos itens que o compõem, e/ ou apoiando iniciativas de APL, é de alta relevância para a construção de uma sociedade econômica e ambientalmente mais saudável, responsável e sustentável.

\section{Referências}

BARBIERI, José Carlos. Desenvolvimento e meio ambiente: as estratégias de mudanças da Agenda 21. 5. ed. Petrópolis: Vozes, 2002.

BELLEN, Hans M. V. Indicadores de sustentabilidade: uma análise comparativa. 2. ed. Rio de Janeiro: FGV, 2006.

BISQUERRA, Rafael; SARRIERA, Jorge C.; MARTÍNEZ, Francesc. Introdução à estatística: enfoque informático com o pacote estatístico SPSS. Porto Alegre: Artmed, 2004.

BOLLINGER, F.P.; NETO SCANDAR, W. J. Estatísticas Ambientais e indicadores de Desenvolvimento Sustentável no Brasil. In: ROMEIRO, Ademar R. (Org.). Avaliação e contabilização de impactos ambientais. Campinas: Ed. UNICAMP: 2004. p. 271-299.

BRAGA, Tânia M. et al. Índices de Sustentabilidade Municipal: o desafio de Mensurar. Nova Economia, Belo Horizonte, v. 14, n. 3, p. 11-13, set./dez., 2004. Disponível em: < http:// www.face.ufmg.br/novaeconomia/sumarios/v14n3/140301.pdf > . Acesso em: 18 jul. 2012. 
CATIZZONE, Márcio. Desenvolvimento sustentável: um conceito que precisa se tornar operacional. In: ROMEIRO, Ademar R. (Org.). Avaliação e contabilização de impactos ambientais. Campinas: Ed. UNICAMP: 2004. p. 216-230.

COSTA, Márcio J. P. Trajetória do desenvolvimento: da ênfase no crescimento econômico às expectativas do desenvolvimento sustentável. 2006. Dissertação (Mestrado em Desenvolvimento e meio ambiente) - Universidade Federal da Alagoas, Maceió, 2006.

. Arranjos produtivos locais: a construção de sua territorialidade no Estado de Alagoas. Maceió: FEAC/UFAL (Projeto de Pesquisa), 2008.

COSTA, Márcio J. P.; LUSTOSA, Maria C. J. Mensuração do desenvolvimento socioeconômico e ambiental. In: ENCONTRO DA SOCIEDADE BRASILEIRA ECOLÓGICA, 7., 2007, Fortaleza. Anais eletrônicos... Brasília: Sociedade Brasileira de Economia Ecológica, 2007. Disponível em: < http://www.ecoeco.org.br/publicacoes/ encontros/111-vii-encontro-nacional-da-ecoeco-fortaleza-ce-2007> . Acesso em: 18 jul. 2012.

ENVIROMMENTAL SUSTAINABILITY INDEX. 2002. Disponível em: < http://www. ciesin.columbia.edu >. Acesso em: 18 jul. 2012.

GOLDMEIER, Valtemir B. Coletânea Gestão Pública Municipal. Brasília: CNM, 2004. v. 9. INSTITUTO BRASILEIRO DE GEOGRAFIA E ESTATÍSTICA (IBGE). Perfil dos municípios brasileiros: meio ambiente 2002. Rio de Janeiro: IBGE, 2005a. 2005b.

. Indicadores de desenvolvimento sustentável. Brasil 2004. Rio de Janeiro: IBGE,

JANNUZZI, Paulo M. Indicadores sociais no Brasil: conceitos, fontes de dados e aplicações. 2. ed. Campinas: Alínea, 2003.

LAGES, Vinícius; TONHOLO, Josealdo (Org.). Desafios de competitividade em Arranjos produtivos locais: dinâmicas de inovação e papel das incubadoras de empresas e parques tecnológicos. Brasília: ANPROTEC, 2006.

LASTRES, Helena et al. (Coord.). Interagir para competir: promoção de Arranjos produtivos e inovativos no Brasil. Brasília: CNPq/FINEP/SEBRAE, 2002.

LUSTOSA, Maria C. Questões ambientais em Arranjos e sistemas produtivos e inovativos locais: referencial teórico, referencial analítico e verificação empírica. Brasília: CNPQ, 2006. (Relatório técnico final)

MARTINS, Ana R.; FERRAZ, Fernando T.; COSTA, Marcio M. Sustentabilidade ambiental como nova dimensão do índice de desenvolvimento humano dos países. Revista do BNDES, Rio de Janeiro, v. 13, n. 26, p. 139-163, dez., 2006. Disponível em: < http://www.bndes.gov.br/SiteBNDES/export/sites/default/bndes_pt/Galerias/Arquivos/ conhecimento/revista/rev2607.pdf > . Aceso em: 18 jul. 2012.

MELO, Ricardo L.; HANSEN, Dean L. (Org.). Desenvolvimento regional e local: novas e velhas questões. São Cristóvão: UFS, 2007.

MENEZES, Luis C. Índice de amadurecimento gerencial. Rio de Janeiro: ENCE, 2006.

OLIVEIRA, Sonia M.; CARVALHO, Paulo G.M.; BARCELLOS, Frederico C. Instrumentos de gestão local e meio ambiente. In: ENCONTRO NACIONAL DA SOCIEDADE 
BRASILEIRA DE ECONOMIA ECOLÓGICA, 5., 2003, Caxias do Sul. Anais eletrônicos... Brasília: Sociedade Brasileira de Economia Ecológica, 2003. Disponível em: < http:// www.ecoeco.org.br/conteudo/publicacoes/encontros/v_en/Mesa3/4.pdf > . Acesso em: 18 jul. 2012.

PHILIPPI JUNIOR, Arlindo et al (Ed.). Municípios e meio ambiente: perspectivas para a municipalização de gestão ambiental no Brasil. São Paulo: ANAMMA, 1999.

PROGRAMA DAS NAÇÕES UNIDAS PARA O DESENVOLVIMENTO. Relatório do desenvolvimento humano 1990. New York: PNUD, 1990. Disponível em: < http://hdr. undp.org/en/reports/global/hdr1990 > .Acesso em: 11 de agosto de 2012. de 2009.

QUIROGA, Rayen M. Indicadores de sustentabilidade ambiental y de desarrollo sostenible: estado del arte y perspectiva. Santiago: CEPAL, 2001. (Série Manuales)

SACHS, Ignacy. Desenvolvimento includente, sustentável e sustentado. Rio de Janeiro: Garamond, 2004.

SINGER, Paul; SOUZA, André R. (Org.). A economia solidária no Brasil: a autogestão como resposta ao desemprego. São Paulo: Contexto, 2000.

VALE, Gláucia M. V. Territórios vitoriosos: o papel das redes organizacionais. Rio de Janeiro: Garamond, 2007.

SCANDER NETO, Wadih J. Síntese que organiza o olhar: uma proposta para construção e representação de indicadores de desenvolvimento sustentável e sua aplicação para os municípios fluminenses. 2006. 110 f. Dissertação. Rio de Janeiro, 2006. Disponível me: < http://www.dominiopublico.gov.br/download/texto/cp079267.pdf:>

Acesso em: 18 jul. 2012.

198 | Nexus Econômicos - CME-UFBA 


\section{Anexo}

ÍNDICE MUNICIPAL DE GESTÃO AMBIENTAL POR COMPONENTES, SEGUNDO OS APL QUE COMPÕEM OS SETORES ECONÔMICOS DO ESTADO DE ALAGOAS.

\begin{tabular}{|c|c|c|c|c|c|c|c|}
\hline$\stackrel{O}{i}$ & $\begin{array}{l}\text { ÍNDICE MUNICIPAL } \\
\text { COMPONENTES, SEGUN } \\
\text { SETORES ECONÔMICO } \\
\text { ACORDO COM A CLAS }\end{array}$ & $\begin{array}{l}\text { E GESTÃO A } \\
\text { O OS APLS } \\
\text { DO ESTADC } \\
\text { IFICAÇÃO }\end{array}$ & $\begin{array}{l}\text { MBII } \\
\text { QUE } \\
\text { DE } \\
\text { OS }\end{array}$ & $\begin{array}{l}\text { NTAI } \\
\text { OM } \\
\text { LAG }\end{array}$ & $\begin{array}{l}\text { OR } \\
\text { EM } \\
\text { AS D } \\
2002\end{array}$ & & 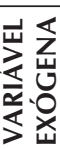 \\
\hline 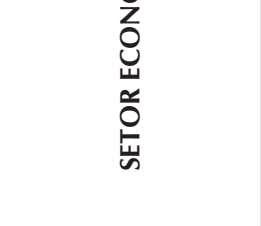 & $\vec{a}$ & 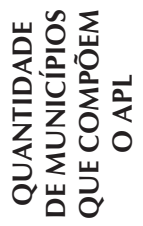 & $\overleftarrow{\underline{0}}$ & 文 & 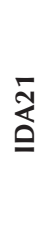 & 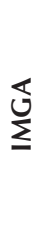 & $\underset{\underline{1}}{\underline{\underline{1}}}$ \\
\hline APICULTURA & APICULTURA NO SERTÃO & 5 & $\begin{array}{l}\tilde{m} \\
\tilde{0}\end{array}$ & $\underset{\substack{\sim \\
\sim}}{\stackrel{0}{0}}$ & $\begin{array}{l}0 \\
\text { nㅏㅇ } \\
0\end{array}$ & $\begin{array}{l}\hat{0} \\
\tilde{n} \\
0\end{array}$ & $\begin{array}{l}\infty \\
\infty \\
12 \\
0\end{array}$ \\
\hline $\begin{array}{l}\text { AQUICULTURA E } \\
\text { PESCA }\end{array}$ & $\begin{array}{l}\text { PISCICULTURA DELTA DO } \\
\text { SÃO FRANCISCO }\end{array}$ & 13 & 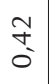 & 웅 & $\stackrel{\stackrel{n}{m}}{0}$ & 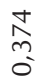 & $\begin{array}{l}0 \\
10 \\
0 \\
0\end{array}$ \\
\hline $\begin{array}{c}\text { LEITE E } \\
\text { DERIVADOS }\end{array}$ & LATICÍNIOS SERTÃO & 8 & $\stackrel{m}{0}$ & $\frac{\infty}{\sigma}$ & $\stackrel{+}{\stackrel{+}{0}}$ & $\begin{array}{c}\text { ț } \\
\text { m } \\
0\end{array}$ & $\begin{array}{l}\infty \\
12 \\
0 \\
0\end{array}$ \\
\hline $\begin{array}{l}\text { MADEIRA E } \\
\text { MÓVEIS }\end{array}$ & MÓVEIS AGRESTE & 2 & $\begin{array}{l}\stackrel{0}{1} \\
\vdots\end{array}$ & $\begin{array}{l}i \\
m \\
0\end{array}$ & $\begin{array}{l}0 \\
\stackrel{1}{10} \\
0\end{array}$ & $\begin{array}{l}8 \\
\substack{0 \\
0 \\
0}\end{array}$ & $\begin{array}{l}\bar{\sigma} \\
\stackrel{0}{0} \\
0\end{array}$ \\
\hline MANDIOCULTURA & MANDIOCA AGRESTE & 12 & $\tilde{m}$ & $\begin{array}{l}\hat{0} \\
0 \\
0\end{array}$ & $\begin{array}{l}0 \\
\vdots \\
0\end{array}$ & 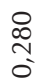 & $\begin{array}{c}\text { ñ } \\
\hat{\vdots} \\
0\end{array}$ \\
\hline $\begin{array}{l}\text { OVINO E } \\
\text { CAPRINO }\end{array}$ & $\begin{array}{c}\text { OVINOCAPRINOCULTURA } \\
\text { SERTÃO }\end{array}$ & 21 & $\frac{6}{5}$ & $\stackrel{\text { Ln }}{5}$ & 웅 & 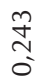 & $\begin{array}{l}\text { Oे } \\
\text { Ln } \\
0\end{array}$ \\
\hline $\begin{array}{l}\text { TECNOLOGIA DA } \\
\text { INFORMAÇÃO }\end{array}$ & $\begin{array}{l}\text { TECNOLOGIA DE } \\
\text { INFORMAÇÃO MACEIÓ }\end{array}$ & 1 & $\stackrel{+}{8}$ & $\begin{array}{l}0 \\
:\end{array}$ & $\begin{array}{l}0 \\
\stackrel{1}{10} \\
0\end{array}$ & $\begin{array}{l}\stackrel{8}{8} \\
\vdots \\
0\end{array}$ & $\begin{array}{l}\hat{\sigma} \\
\hat{N} \\
0\end{array}$ \\
\hline
\end{tabular}




\begin{tabular}{|c|c|c|c|c|c|c|c|}
\hline \multirow{3}{*}{ TURISMO } & $\begin{array}{c}\text { TURISMO NA COSTA DOS } \\
\text { CORAIS }\end{array}$ & 8 & $\begin{array}{l}+ \\
0 \\
0\end{array}$ & $\stackrel{\stackrel{L}{N}}{0}$ & 只 & $\begin{array}{l}\text { } \\
\text { ñ } \\
0\end{array}$ & $\begin{array}{l}\bar{\sigma} \\
\stackrel{0}{0} \\
0\end{array}$ \\
\hline & $\begin{array}{l}\text { TURISMO LAGOAS E } \\
\text { MARES DO SUL }\end{array}$ & 10 & $\begin{array}{l}\stackrel{2}{1} \\
0\end{array}$ & $\begin{array}{l}0 \\
\text { ำ } \\
0\end{array}$ & 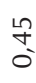 & 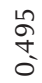 & $\begin{array}{l}\text { J } \\
\stackrel{1}{1} \\
0\end{array}$ \\
\hline & $\begin{array}{c}\text { TURISMO CAMINHOS DO } \\
\text { SÃO FRANCISCO }\end{array}$ & 12 & 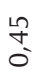 & 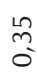 & $\underset{f}{\stackrel{f}{0}}$ & $\begin{array}{c}\hat{\sigma} \\
\tilde{N} \\
0\end{array}$ & 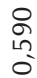 \\
\hline
\end{tabular}

Fonte: Elaboração própria.

Observação: os valores dos índices correspondem às suas respectivas médias por APL. 